\title{
ON THE VON NEUMANN ALGEBRA OF AN ERGODIC GROUP ACTION ${ }^{1}$
}

ROBERT J. ZIMMER

\begin{abstract}
We give a criterion that an ergodic action be amenable in terms of the operator algebra associated to it by the Murray-von Neumann construction.
\end{abstract}

The notion of an amenable ergodic action of a locally compact group was introduced by the author in [7]. (The reader is cautioned that this is not the same as the notion introduced by Greenleaf in [3]; see [7] for a discussion of this point.) In this note, we give a criterion for an action of a countable discrete group to be amenable in terms of the von Neumann algebra associated to it by the classical Murray-von Neumann construction.

We recall that J. T. Schwartz has introduced the following property of von Neumann algebras [6, p. 168]. If $A$ is a von Neumann algebra on a Hilbert space $H, A$ is said to have property $\mathrm{P}$ if for any $T \in B(H)$, the closed (weak operator topology) convex hull of $\left\{U^{*} T U \mid U \in U(A)\right\}$ contains an element of $A^{\prime}$, where $U(A)$ is the unitary group of $A$, and $A^{\prime}$ is, as usual, the commutant of $A$. If $G$ is a countable discrete group and $R(G)$ the von Neumann algebra generated by the right regular representation of $G$, then $R(G)$ has property $\mathrm{P}$ is and only if $G$ is amenable [5, Proposition 4.4.21]. More generally, Schwartz shows that if $G$ is amenable and acts ergodically on a Lebesgue space $(S, \mu)$, then $R(S \times G)$, the algebra of the Murray-von Neumann construction (described below) has property $P$ [6, p. 198]. Conversely, if $R(S \times G)$ has property $\mathrm{P}$ and $\mu$ is finite and $G$-invariant, then $G$ must be amenable [6, p. 200]. The point of this note is to prove a stronger converse, without the assumption of a finite invariant measure. Namely, we show that if $R(S \times G)$ has property $\mathrm{P}$, then $S$ is an amenable $G$-space (definition below). The result quoted above in the case of finite invariant measure then follows from [7, Proposition 4.4], which asserts that if $S$ is an amenable $G$-space with finite invariant measure (or mean), then $G$ itself must be amenable.

We recall the Murray-von Neumann construction. Let $S$ be a standard Borel space, $G$ a countable discrete group with a right Borel action of $G$ on $S$. We suppose $\mu$ is a probability measure on $S$ quasi-invariant and ergodic

Received by the editors August 30, 1976 and, in revised form, February 20, 1977. AMS (MOS) subject classifications (1970). Primary 28A65, 46L10.

${ }^{1}$ Research supported by the Naval Academy Research Council.

๑ American Mathematical Society 1977 
under $G$. We let $r(s, g)$ be the Radon-Nikodym cocycle of the action, i.e. a Borel function such that $d \mu(s g)=r(s, g) d \mu(s)$. Let $U_{g}: L^{2}(G) \rightarrow L^{2}(G)$ be the right regular representation of $G$, and define a unitary representation $\tilde{U}_{g}$ : $L^{2}(S \times G) \rightarrow L^{2}(S \times G)$ by

$$
\left(\tilde{U}_{g} f\right)(s, h)=f(s g, h g) r(s, g)^{1 / 2} \text {. }
$$

Here $S \times G$ has the product measure of $\mu$ with Haar measure. Let $V_{g}$ be the left regular representation of $G$ on $L^{2}(G)$ and define $\tilde{V}_{g}: L^{2}(S \times G) \rightarrow L^{2}(S$ $\times G)$ by

$$
\left(\tilde{V}_{g} f\right)(s, h)=f\left(s, g^{-1} h\right) \text {. }
$$

If $f \in L^{\infty}(S)$, then $f$ defines a multiplication operator $M_{f}$ on $L^{2}(S \times G)$ by $\left(M_{f} h\right)(s, g)=f(s) h(s, g)$ and a multiplication operator $N_{f}$ by $\left(N_{f} h\right)(s, g)=$ $f(s g) h(s, g)$. We let $L$ be the von Neumann algebra generated by $\left\{\tilde{V}_{g}, N_{f}\right\}$ and $R$ the von Neumann algebra generated by $\left\{\tilde{U}_{g}, M_{f}\right\}$. Then $R^{\prime}=L$, and there is a unitary involution $J$ on $L^{2}(S \times G)$ such that $J R J=L[1$, pp. 137-138]. Thus $R$ and $L$ are spatially isomorphic and one will have property $P$ if and only if the other does.

The definition of an amenable ergodic action given in [7] is based upon an "invariant section property" and is motivated by the virtual subgroup viewpoint of Mackey. We review the definition. Suppose $E$ is a separable Banach space and $\gamma: S \times G \rightarrow \operatorname{Iso}(E)$ is a Borel cocycle, where $\operatorname{Iso}(E)$ is the group of isometric isomorphisms of $E$ with the Borel structure of the strong operator topology. Let $E_{1}^{*}$ be the unit ball in the dual of $E$ with the $\sigma\left(E^{*}, E\right)$ topology. Then there is an induced adjoint cocycle $\gamma^{*}: S \times G \rightarrow \operatorname{Homeo}\left(E_{1}^{*}\right)$, $\gamma^{*}(s, g)=\left(\gamma(s, g)^{*}\right)^{-1}$. A Borel function $\phi: S \rightarrow E_{1}^{*}$ is called an invariant section for $\gamma$ if for each $g \in G, \gamma^{*}(s, g) \phi(s g)=\phi(s)$ for almost all $s \in S$. Now suppose that for each $s, A_{s} \subset E_{1}^{*}$ is a compact convex set, that $\left\{(s, x) \mid x \in A_{s}\right\}$ is a Borel subset of $S \times E_{1}^{*}$, and that for each $g, \gamma^{*}(s, g) A_{s g}$ $=A_{s}$ for almost all $s$. Then $S$ is called an amenable $G$ space if for each such cocycle $\gamma$ and each such collection $\left\{A_{s}\right\}$, there is an invariant section $\phi$ with $\phi(s) \in A_{s}$ for almost all $s$. In [7] it is shown that any ergodic action of any amenable group is amenable, but that nonamenable groups can also have amenable actions. For example the range-closure of a cocycle [4] of an amenable action into any locally compact group is amenable [7, Theorem 3.3].

THEOREM. If $G$ is a countable discrete group acting ergodically on $(S, \mu)$ and the von Neumann algebra $R$ (or equivalently, $L$ ) has property $\mathrm{P}$, then $S$ is an amenable $G$-space. (Here $\mu$ is a quasi-invariant probability measure.)

Proof. If $f \in L^{2}(S \times G)$, define $f_{s}(g)=f(s g, g) r(s, g)^{1 / 2}$. Then $f_{s} \in$ $L^{2}(G)$ for almost all $s$, and a straightforward calculation shows that $f \rightarrow$ $\int^{\oplus} f_{s} d \mu$ is a unitary isomorphism of $L^{2}(S \times G) \cong \int_{s}^{\oplus} L^{2}(G)$. One further readily verifies that under this isomorphism, $\tilde{U}_{g}$ corresponds to $\int_{s}^{\oplus} U_{g}$ and that if $T \in B\left(L^{2}(S \times G)\right)$ corresponds to the decomposable operator $\int^{\oplus} T_{s}$ in 
$B\left(\int_{s}^{\oplus} L^{2}(G)\right)$, then $T$ commutes with $\tilde{V}_{g}$ if and only if for each $g, V_{g}^{-1} T_{s} V_{g}=$ $T_{s g}$ for almost all $s$. Since the operators $\tilde{U}_{g}$ and $M_{f}, f \in L^{\infty}(S)$, correspond to decomposable operators, it follows that every element of $R$ is decomposable with respect to this direct integral decomposition of $L^{2}(S \times G)$.

Now suppose that $L$ has property P. Then [5, Proposition 4.4.15] there is a linear mapping $P: B\left(L^{2}(S \times G)\right) \rightarrow R\left(=L^{\prime}\right)$ such that

(i) $\|P\| \leqslant 1, P(I)=I, T \geqslant 0$ implies $P(T) \geqslant 0$.

(ii) $P(T) \in C(T)$, where $C(T)$ is the closed convex hull of $\left\{U T U^{*} \mid U \in\right.$ $U(L)\}$.

(iii) $P\left(S_{1} T S_{2}\right)=S_{1} P(T) S_{2}$ if $S_{1}, S_{2} \in R$.

If $f \in L^{\infty}(S \times G)$, we have the multiplication operator $M_{f} \in B\left(L^{2}(S \times\right.$ $G)$ ), and since each element of $R$ is decomposable, we can write $P\left(M_{f}\right)=$ $\int^{\oplus} T_{s}^{f} d \mu$. For $f \in L^{\infty}(S \times G)$, write $(f \cdot g)(s, h)=f(s g, h g)$. Then $\tilde{U}_{g} P\left(M_{f}\right) \tilde{U}_{g}^{-1}=P\left(\tilde{U}_{g} M_{f} \tilde{U}_{g}^{-1}\right)=P\left(M_{f \cdot g}\right)$. Thus $\tilde{U}_{g}\left(\int^{\oplus} T_{s}^{f}\right) \tilde{U}_{g}^{-1}=\int^{\oplus} T_{s}^{f \cdot g}$, i.e. $\int^{\oplus} U_{g} T_{s}^{f} U_{g}^{-1}=\int^{\oplus} T_{s}^{f \cdot g}$. It follows that for each $g, U_{g} T_{s}^{f} U_{g}^{-1}=T_{s}^{f \cdot g}$ for almost all $s$. We also note that since $\int^{\oplus} T_{s}^{f} \in L^{\prime}$, it follows from the remarks in the preceding paragraph that for each $g, V_{g}^{-1} T_{s}^{f} V_{g}=T_{s g}^{f}$ a.e.

For $f \in L^{\infty}(S \times G)$, define $\sigma(f)(s)=\left\langle T_{s}^{f} \chi_{e} \mid \chi_{e}\right\rangle$ where $\chi_{e} \in L^{2}(G)$ is the characteristic function of the identity. Then $\sigma: L^{\infty}(S \times G) \rightarrow L^{\infty}(S)$ and this map has the following properties:

(i) $\|\sigma(f)\|_{\infty} \leqslant\|f\|_{\infty}$.

(ii) $\sigma(1)=1$.

(iii) If $f \geqslant 0, \sigma(f) \geqslant 0$.

(iv) If $A \subset S$ is measurable, $\sigma\left(f \chi_{A \times G}\right)=\sigma(f) \chi_{A}$.

(v) $\sigma(f \cdot g)=\sigma(f) \cdot g$.

Properties (i)-(iv) follow in a straightforward manner from the properties of the map $P$ listed above and some elementary properties of direct integrals of operators. To see (v), note that for each $g$ and almost all $s$,

$$
\begin{aligned}
\sigma(f \cdot g)(s) & =\left\langle T_{s}^{f \cdot g} \chi_{e} \mid \chi_{e}\right\rangle=\left\langle U_{g} T_{s}^{f} U_{g}^{-1} \chi_{e} \mid \chi_{e}\right\rangle \\
& =\left\langle U_{g} V_{g} T_{s g}^{f} V_{g}^{-1} U_{g}^{-1} \chi_{e} \mid \chi_{e}\right\rangle=\left\langle T_{s g}^{f} V_{g}^{-1} U_{g}^{-1} \chi_{e} \mid V_{g}^{-1} U_{g}^{-1} \chi_{e}\right\rangle \\
& =\left\langle T_{s g}^{f} \chi_{e} \mid \chi_{e}\right\rangle=\sigma(f)(s g) .
\end{aligned}
$$

We now demonstrate how the map $\sigma$ can be used to show that $S$ is amenable. Suppose $E, \gamma$, and $\left\{A_{s}\right\}$ are as in the discussion preceding the statement of the theorem. Since $\left\{\left(s, A_{s}\right)\right\}$ is Borel, there is a measurable function $b: S \rightarrow E_{1}^{*}$ such that $b(s) \in A_{s}$ for almost all $s \in S$. Define $F$ : $S \times G \rightarrow E_{1}^{*}$ by $F(s, g)=\gamma^{*}\left(s, g^{-1}\right) b\left(s g^{-1}\right)$. Then for each $\theta \in E,(s, g) \rightarrow$ $\langle\theta, F(s, g)\rangle$ is in $L^{\infty}(S \times G)$ (where $\langle$,$\rangle is the duality of E$ and $\left.E^{*}\right)$, and the map $E \rightarrow L^{\infty}(S), \theta \rightarrow \sigma(\langle\theta, F(s, g)\rangle)$ is linear with norm $\leqslant 1$. It follows from [2, p. 582] that there is a measurable function $a: S \rightarrow E_{1}^{*}$ such that $\sigma(\langle\theta, F(s, g)\rangle)(s)=\langle\theta, a(s)\rangle$ a.e. We claim that $a(s)$ is the required invariant section.

LEMMA. For all essentially bounded and measurable $\theta: S \rightarrow E$, 


$$
\sigma(\langle\theta(s), F(s, g)\rangle)(s)=\langle\theta(s), a(s)\rangle \text { a.e. }
$$

Proof. Suppose first that $\theta$ is a simple function, i.e. $\theta(s)=\Sigma_{1}^{\infty} \theta_{i} \chi_{A_{i}}(s)$ where $\left\{A_{i}\right\}$ is a countable partition of $S$ and $\theta_{i} \in E$. Fix $j$. Then for almost all $s \in A_{j}$, by property (iv) of $\sigma$,

$$
\begin{aligned}
\sigma(\langle\theta(s), F(s, g)\rangle)(s) & =\sigma\left(\langle\theta(s), F(s, g)\rangle \chi_{A_{j} \times G}\right)=\sigma\left(\left\langle\theta_{j}, F(s, g)\right\rangle \chi_{A_{j} \times G}\right) \\
& =\sigma\left(\left\langle\theta_{j}, F(s, g)\right\rangle\right)(s)=\left\langle\theta_{j}, a(s)\right\rangle=\langle\theta(s), a(s)\rangle .
\end{aligned}
$$

Since $j$ is arbitrary, this lemma holds for simple $\theta$. If $\theta$ is arbitrary, then there are simple functions $\theta_{n}$ with $\left\|\theta_{n}-\theta\right\|_{\infty} \rightarrow 0$ by virtue of the separability of $E$. Since $F(s, g) \in E_{1}^{*},\left\langle\theta_{n}(s), F(s, g)\right\rangle \rightarrow\langle\theta(s), F(s, g)\rangle$ in \|\|$_{\infty}$ on $S \times G$, and by the norm continuity of $\sigma$,

$$
\sigma\left(\left\langle\theta_{n}(s), F(s, g)\right\rangle\right) \rightarrow \sigma(\langle\theta(s), F(s, g)\rangle)
$$

in $L^{\infty}(S)$. Clearly $\left\langle\theta_{n}(s), a(s)\right\rangle \rightarrow\langle\theta(s), a(s)\rangle$ a.e., and the lemma follows.

Corollary. Suppose $\alpha: S \rightarrow \operatorname{Iso}(E)$ is measurable. Then for all $\theta \in E$,

$$
\sigma\left(\left\langle\theta, \alpha(s)^{*} F(s, g)\right\rangle\right)=\left\langle\theta, \alpha(s)^{*} a(s)\right\rangle .
$$

Proof. This is equivalent to $\sigma(\langle\alpha(s) \theta, F(s, g)\rangle)=\langle\alpha(s) \theta, a(s)\rangle$ which holds by the lemma.

We now show that $a(s)$ is an invariant section. Suppose $h \in G$. Then by property (v) of $\sigma$,

$$
\sigma(\langle\theta, F(s, g)\rangle \cdot h)(s)=\langle\theta, a(s)\rangle \cdot h=\langle\theta, a(s h)\rangle .
$$

But the first term of this equation

$$
\begin{aligned}
& =\sigma\left(\left\langle\theta, \gamma^{*}\left(s h, h^{-1} g^{-1}\right) b\left(s g^{-1}\right)\right\rangle\right)(s) \\
& =\sigma\left(\left\langle\theta, \gamma^{*}(s, h)^{-1} \gamma^{*}\left(s, g^{-1}\right) b\left(s g^{-1}\right)\right\rangle\right)(s)
\end{aligned}
$$

and by the corollary, since $\gamma^{*}(s, h)$ is the adjoint of an isometric isomorphism, this $=\left\langle\theta, \gamma^{*}(s, h)^{-1} a(s)\right\rangle$. Since $E$ is separable, it follows [2, Theorem 8.17.2(c)] that $\gamma^{*}(s, h)^{-1} a(s)=a(s h)$ for almost all $s$, i.e. $a(s)$ is an invariant section.

Thus, to complete the proof it suffices to show $a(s) \in A_{s}$ for almost all $s$. Let $\left\{\theta_{i}\right\}$ be a countable dense subset of $E$, considered as linear functionals on $E^{*}$. Then the hyperplanes in $E^{*}$ defined by $\theta_{i}=q, q$ rational, separate all compact convex subsets of $E_{1}^{*}$ from points in $E_{1}^{*}$. Therefore, it suffices to show that for all $\theta$ and $q, \theta\left(A_{s}\right) \geqslant q$ implies $\theta(a(s)) \geqslant q$ for almost all $s$. Given $\theta$ and $q$, let $S_{0}=\left\{s \in S \mid \theta\left(A_{s}\right) \geqslant q\right\}$. Then $S_{0}$ is measurable by [7, Lemma 1.7]. Suppose $\mu\left(S_{0}\right)>0$. Then by property (iii) of $\sigma$,

$$
\sigma\left(\langle\theta, F(s, g)\rangle \chi_{s_{0} \times G}\right) \geqslant \sigma\left(q \cdot \chi_{s_{0} \times G}\right)=q \chi_{s_{0}} .
$$

Thus $\langle\theta, a(s)\rangle \cdot \chi_{s_{0}} \geqslant q \chi_{s_{0}}$, so $\theta(a(s)) \geqslant q$ for almost all $s \in S_{0}$. Since $\theta$ and $q$ are arbitrary, the theorem follows. 


\section{REFERENCES}

1. J. Dixmier, Les algèbres d'opérateurs dans l'espace Hilbertien, Gauthier-Villars, Paris, 1969.

2. R. E. Edwards, Functional analysis, Holt, Rinehart and Winston, New York, 1965.

3. F. P. Greenleaf, Amenable actions of locally compact groups, J. Functional Anal. 4 (1969), 295-315.

4. G. W. Mackey, Ergodic theory and virtual groups, Math. Ann. 166 (1966), 187-207.

5. S. Sakai, $C^{*}$-algebras and $W^{*}$-algebras, Springer-Verlag, New York, 1971.

6. J. T. Schwartz, $W^{*}$-algebras, Gordon and Breach, New York, 1967.

7. R. J. Zimmer, Amenable ergodic group actions and an application to Poisson boundaries of random walks, J. Functional Analysis (to appear).

Department of Mathematics, U. S. Naval ACademy, Annapolis, Maryland 21402

Current address: Department of Mathematics, University of Chicago, Chicago, Illinois 60637 